\title{
SYSTEM TO CONVERT 2-D X-RAY IMAGE INTO 3-D X-RAY IMAGE IN DENTISTRY
}

\author{
Trupti Sardar', Aniket Bhawkar ${ }^{2}$ \\ ${ }^{I}$ Department of Computer Engineering, VIIT, Maharashtra, India \\ ${ }^{2}$ Department of Information Technology, VIIT, Maharashtra, India
}

\begin{abstract}
An X-Ray machine is used to capture the formation of bones and tissues by sending X-Ray particles through the body. Dental XRays help dentists envision infections of the teeth and adjacent tissue that cannot be seen with a simple oral test. In addition, $X$ Rays help the dentist discovery and treat dental complications early in their growth. But not all diseases can be detected by a 2-D $X$-Ray. This system is proposed to get a 3-D X-Ray image of the infected area and give the dentist more precise expression based on patient anatomy. This technology is unique for a widespread variety of specialties, with a broad range of imaging views for analysis, preparation, case presentation, and cure for the dentist whose practice includes oral surgery, general dentistry, implants, orthodontics and other specialties. We focus on new system which can convert 2-D Dental X-Ray to 3-D Dental X-Ray using the proposed architecture and some needful hardware.
\end{abstract}

Keywords: 2-D to 3-D X-Ray, Dentistry, Dental Complications, Diagnosis and Treatment

\section{INTRODUCTION}

X-Ray examinations are commonly done to display bones and certain other tissues. Dental X-Rays are images of the teeth, bones, and soft tissues around them to help find complications in the scanned area. 2-D X-Ray images can show cavities, hidden dental structures (such as wisdom tooth), and bone loss that cannot be seen during a visual examination. The disadvantage of 2-D X-Rays is that they do not give detailed descriptions about the scanned area. But at the same time MRI or CT scans are exceptional tools; as CT scan is capable of creating a 3-D image of bone structures, facilitating a doctor while an X-Ray creates a 2-D image. The advantage of 2-D X-Rays is that they are cheaper in price than that of similar medical procedures. If a patient requires a medical scan, 2-D X-Ray costs less than that of a MRI scan or a CT scan. X-Ray machine are by itself cheaper for a hospital or a doctor's office or a dentist to maintain than any similar operational tools. There are also machines which capture 3-D X-Ray images but they are quite expensive, which all medical experts cannot afford. Also it is expensive for the patients.

The proposed system supports both dentist and patients who want the treatment to be done in low cost and also in efficient way. For this research of 2-D X-Ray to 3-D X-Ray conversion, we focus on software which can convert 2-D Dental X-Ray to 3-D Dental X-Ray image with the use of some needful hardware.

\section{LITERATURE SURVEY}

This section includes the work done on related topics by various researchers. Following is the brief description of some of them:
Mizutani R et al. [1], proposed a paper to process a highresolution X-Ray micro-tomography which has been provided in practical approach to convert it into a three dimensional (3-D) image. This approach is useful in determining the various bone structure and the arrangement of tissues in three dimensions. Examples of microtomographic studies are overviewed to present an outline of biological applications of X-ray micro-tomography. We also provide perspectives of biological micro-tomography as the convergence of sciences in X-ray optics, biology, and structural analysis.

Phan et al. [2], proposed a paper on 2-D to 3-D image conversion in which segmentation algorithms are used to generate a final cohesive depth map. The obtained results demonstration that we can produce good quality stereoscopic image pairs. This image pairs can be used in defining the 2-D to 3-D image conversion in the proposed system. This paper also proposed a study about Image Stability.

Sneha. K et al. [3], proposed a paper in which 2-D images can be converted into 3-D images. This paper resolves the main challenge of 3-D imaging that is the noise content of the input image that degraded quality of the reconstructed image. In this technique after the standardization, the image it is filtered to reduce the noise content. The proposed system is efficient in reconstructing the image and it ensures a clear boundary distinction and proper three dimensional view of the object.

Park et al. [4] presented an image refining technique for generating accurate 3-D images on computer of the respective object. This technology can be used to achieve perfection on the 3-D X-Ray obtained. 
H. Murata et al. [5] proposed a paper in which three different methods are suggested to convert 2-D image to 3D. In first principle we are able to convert a moving 2-D image into 3-D image. In second method (CID) we are able to convert a stable 2-D image into 3-D using two projections. While the third method is a combination of the earlier two methods for converting 2-D image into 3-D image in Real-Time.

Advantages of proposed system:

- Low cost

- Simple to use

- $\quad$ Refined images

- $\quad$ Easy for diagnosis

\section{PROPOSED METHODOLOGY}

This system is proposed to provide the Dentist with a 3-D $\mathrm{X}$-Ray image of the patients infected oral area. The dentist is supposed to capture a 2-D X-Ray image of the patient's infected oral area with the help of X-Ray camera. The system provides a 3-D X-Ray image so the dentist can detect the intensity of the disease at the early stage. The most important part of this system is a need of 2-D to 3-D image conversion software. Also there is need of 3-D image refining system to define maximum accuracy. Hence the following modules act as the building blocks for the proposed system.

The modules required are:

- 2-D Image Capturing Module

- 2-D to 3-D Image Conversion Module.

- 3-D Image Refining Module.

- 3-D X-Ray Representation Module

Also there is a need of a database to store the 3-D-Xrays of all the patients for further reference if required

Hence the database required is:

- 3-D Image Database

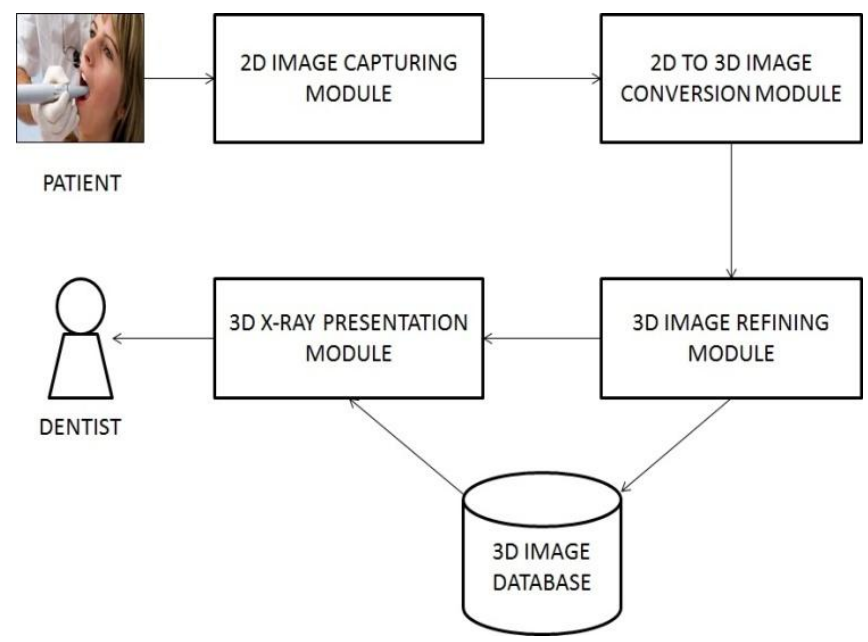

Fig-1: Proposed System Architecture

\section{1 2-D Image Capturing Module}

Many infections of the oral fissure (which contains the teeth and adjacent tissues) are not noticeable when the dentist examines mouth. Dentist is supposed to capture the X-ray of the infected region. Taking the proper X-Ray needs inordinate skills. One must recognize how the vertical and horizontal angle and the time when image is captured can affect the concluding X-Ray. In this system we require two images of the infected area. One would be the top view and the second will be the side view of the tooth. The top view (which can be image or X-ray) is necessary for the width of tooth. We also need the side view (which is X-Ray of tooth) which will describe the height and breadth of tooth.Fig.-2 shows the side view of the tooth. This side view will show how deep the cavity or carries are present and the top view will show the area infected.

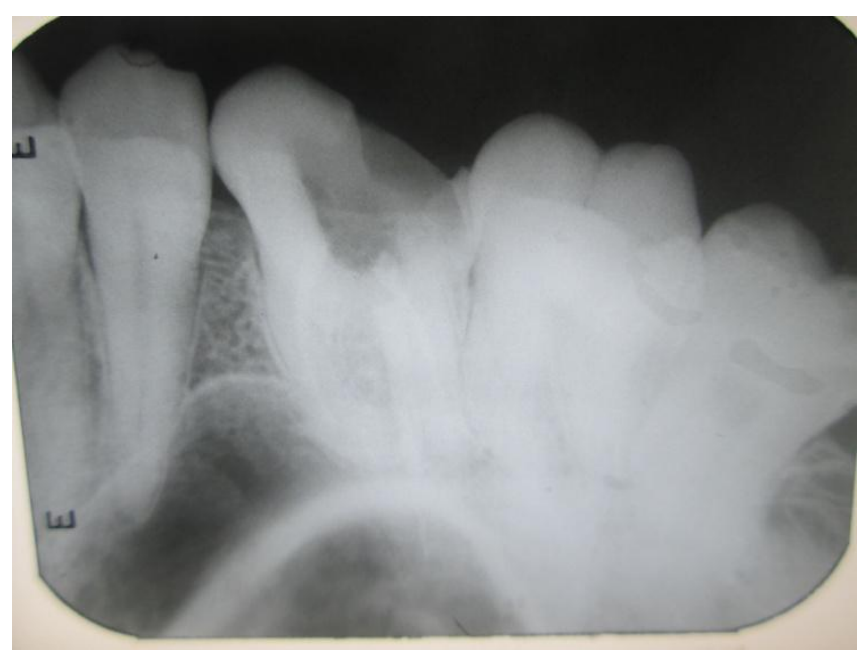

Fig -2: X-Ray of the tooth (Side View)

\subsection{2-D to 3-D Image Conversion Module}

This is the most important module of the system which will help us to get the 3-D image of the tooth. In this we use the technique to convert 2-D image to 3-D.The Computer Image depth technology is used to convert a 2-D stagnant image to 3-D image. The "Computed Image Depth method" is recommended for transforming from all categories of 2-D $\mathrm{X}$-Ray images into 3-D images. The 3-D images are engendered by first calculating the depth of each alienated area of the 2-D images with their divergence and sharpness. The quality of near images is more as compared to far images so we use this technique for the conversion of 2-D $\mathrm{X}$-Ray images to 3-D. The sharpness of the image is exactly opposite to the distance of camera. But in this case the surface of tooth is not uniform. Hence when image is captured there is ambiguousness in the image. The converted image can also be ambiguous. So the Computed Image Depth is implemented into a single-chip LSI with the "Modified Time Difference method". This is the automatic conversion technique by using the two methods that is Modified Time Difference and computed image depth method. So, we use the 2-D to 3-D image translation method 
with this newly established LSI comprehends to generate 3D images.
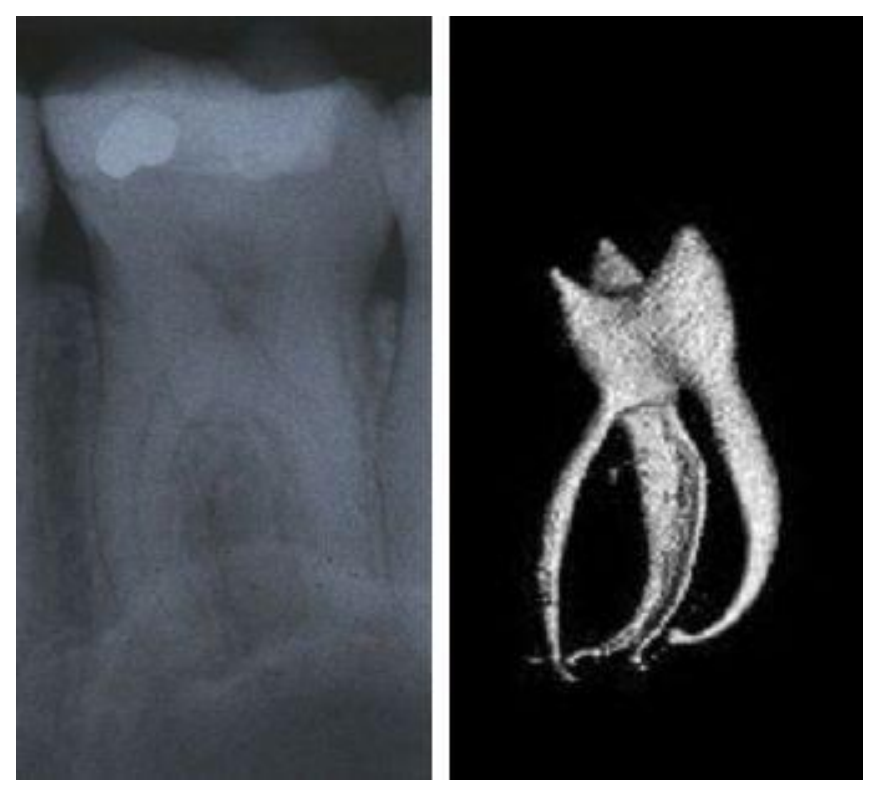

Fig -3: 3-D X-Ray Image of Tooth

\subsection{3-D Image Refining Module}

The 2-D image is been converted to 3-D image. But there may be the chances that the image is not clear. The clearness of the image is the important factor as the diagnosis should be accurate. This module helps to sharpen up the image so as to improve it. As the medical reports should be always accurate this module is important for the system. The 3-D image obtained from this system can experience geometric alterations on reconstructed 3-D surfaces due to the matching problems such as obstructions or mismatching of 2 images. Histogram equalization is an appropriate method to enhance the image. The final enhanced image is little noisy, but it gives typical X-Ray images when their gray scale is expanded. It is advantageous to accentuate the contribution to enhancement made by high-frequency component. If we multiply a high pass filter function by an constant an add on offset so that the zero frequency term is not deleted by the filter. This is called higher-frequency emphasis, given by:

$$
H_{h f e}(u, v)=a+b H_{h p}(u, v)
$$

Where, $a>0=$ and $b>a$

The result obtained using a combination of high frequency emphasis and histogram equalization is superior to the result that would be obtained by using either method alone.

\subsection{3-D X-Ray Presentation Module}

The 3-D X-Ray image received from the above mentioned modules is presented to the dentist using the 3-D XRay Presentation Module. This module provides the dentist with the X-Ray image of the infected area, which can be adjusted as the comfort of the dentist i.e. zoom in, zoom out, change angle of projection and so on. The Dentist get a first eye on the X-Ray in 3-D projection with high level of accuracy which helps him to cure the infected area with high level of perfection.

\subsection{3-D Image Database}

Case history of any patient is the important attire for doctors. Since case history is essential to recommend any further treatment for the patient. Hence, we introduce the 3D image database. The 3-D Images acquired from the 3-D Image Refining Module are saved in the 3-D Image Database for further verification if required by the dentist. This module captures the image from the 3-D Image Refining Module and save the image along with the patients name, proposed infection, area of oral infection, date of $\mathrm{X}$ Ray taken and other miscellaneous data which may be required. The dentist can view any information stored in this database whenever case study is required.

\section{CONCLUSIONS AND FUTURE SCOPE}

The proposed system of converting 2-D X-Ray image into a well refined 3-D X-ray image uses some simple procedures which are followed in Digital Image Processing. The proposed system is capable to demonstrate a 3-D image of a 2-D X-Ray with the help of the mentioned system architecture and some needful hardware devices. For the processing of the system we require two X-Ray images of the tooth to convert it into 3-D image. We also use image refining technique to get the accurate image. The proposed system helps the dentist to adjust the image according to their convenience. Our future work will include implementation of this technology and this technique can also be used to attain 3-D X-Ray model of all the bones or tissues.

\section{ACKNOWLEDGEMENTS}

We take this opportunity to thank people who lend a hand to us for nourishing this system. We express deep gratitude towards Prof. Mrs. J.V. Bagade, for their productive \& hopeful suggestions. We sincerely thank Dr. Preeti Sardar for all the valuable information in the medical field and their encouragement for the project. We also thank all Teaching and Non-teaching staff of VIIT, Pune for their kind of cooperation during our course. The blessings and support of our family and friends has always given us success, we are extremely thankful for their love.

\section{REFERENCES}

[1] Mizutani, R; Suzuki, Y (2012). "X-ray microtomography in biology". Micron (Oxford, England : 1993) 43 (2-3): 104-15.

[2] Phan, Rzeszutek, Androutsos (22-27 May 2011), "Semi-automatic 2-D to 3-D image conversion using a hybrid Random Walks and graph cuts based approach", Acoustics, Speech and Signal Processing (ICASSP), 2011 IEEE International Conference, pp. $897-900$.

[3] Sneha K., T. Sheetal (January 2013), "2-D to 3-D Conversion for Images using Hough Transform", 
International Conference on Information System and Computing (ICISC-2013), pp. $482-486$.

[4] Park, S.-Y., Subbarao, M (6-10 October 2003), “A range image refinement technique for multi-view 3-D model reconstruction", 3-D Digital Imagining and Modeling, 2003. 3-DIM 2003. Proceedings. Fourth International Conference, pp. $147-154$.

[5] H. Murata, X Mori, S. Yamashita, A. Maenaka, S. Okada, K. Oyamada, S. Kishimoto Sanyo Electric Co., Ltd., Osaka, Japan, (1998), “A Real-Time 2-D to 3-D Image Conversion Technique Using Computed Image Depth ", SID Symposium Digest of Technical Papers, Vol. 29, Issue 1, pp. 919 - 923.

[6] X-Ray Micro-tomography at the US National Library of Medicine Medical Subject Headings (MeSH)

[7] Alon Geri, VP Engineering, Co-Founder (05/19/2014), Surgical Theater Offers 3-D Simulations of Medical Procedures, MDT Medical Design Technology.

[8] Digital Image Processing (Second edition) Rafael C. Gonzalez and Richard E. Woods

\section{BIOGRAPHIES}

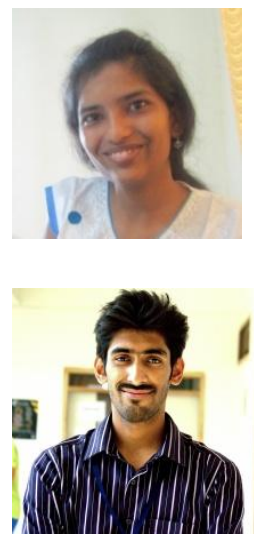

Ms. Trupti Sardar is currently pursuing Bachelor of Engineering in Computer Sciences from University of Pune. Her research interests are Computer Graphics and Digital Image Processing.

Mr. Aniket Bhawkar is currently pursuing Bachelor of Engineering in Information Technology from University of Pune. His research interests are Software Architecture. 